\title{
A Design of Adaptive Virtual Camera View Modes Based on Optimized Virtual Assembly Relation Model
}

\author{
Rui Yang ${ }^{1}$ and WenJie Fan ${ }^{2}$ \\ China Meteorological Administration Training Centre, Beijing, P.R. China \\ ${ }^{1}$ yangr@cma.gov.cn, 2fanwj@cma.gov.cn
}

\begin{abstract}
This paper proposes an optimized virtual assembly relation model on the basis of deep analyses of traditional ones. Some typical adaptive virtual camera view modes are designed based on constraint conditions and dynamic data structure of optimized model mentioned above for a reversible virtual process of assembly and disassembly. These newly designed viewing modes are able to auto-adjust focus and field of view follow and aid user operations by ensuring that camera view continuøusly focuses on virtual operating space currently in need, which lower the difficuty of user operations and heighten the efficiency of virtual operations.

Keywords: Adaptive virtual camera view modes; optimized wirmal assembly relation model; virtual assembly; virtual reality

\section{Introduction}

As we all known, virtual camera view plays an important role in virtual assembly[1-6] since it directly affects player expenence and operation efficiency. It works as the users' point-of-view through which users can expefience the virtual environment and get direct feedback on their operations. However it has been ignored in previous studies[12-16]. Virtual assembly, a typicalapplication of virtual reality technology in industrial assembly, usually takes complicated instruments with massive parts as operation objects. Its operation is not just a single top-down or bottom-up process. Forward and backward operations are able to proceed in parallel, switched freely according to users' intention and interacion. These switch processes are some kind of random choices, which make related camera view controlbecome more complex and uncontrollable. In the general design, the camera view is usually controlled directly by users. It is difficult for the most users who do not have any virtual experience to directly control the camera view. They have to face large quantity of switch processes and precise matching of small parts, which is more likely to occur out of camera view. Missing currently operated parts in the field of view greatly reduces good user experience and operational efficiency.

This paper proposes an optimized virtual assembly relation model and several typical aaptive virtual camera view modes on the basis of deep analyses of previous stadies[7-11]. It aims to define an optimized virtual assembly relation model by a rational set of base object. This optimized model helps us to control the camera view dynamically and efficiently according to current assembly sequences. One of its main advantages is that the view continues to follow and display the user's intention by dynamically setting parameters of camera such as focus and radius. The reasonable constraints and guidance of camera view is significant to ensure that reversible virtual process of assembly and disassembly proceeds smoothly and efficiently. Finally, a user evaluation is conducted and its result is discussed in Chapter 6. It shows that the adaptive camera view modes have a positive impact on the uses' performance with great feedback from them.
\end{abstract}




\section{Traditional Virtual Assembly Relation Model}

Virtual assembly relation model is essential to adaptive control of camera view. The traditional models usually involve three common interpretation methods, namely hierarchy method, relation method and hybrid method. Hierarchy method does well in definitely describing subordinate relationship among parts and subassemblies. Relation method is good at comprehensively presenting various complex assembly relations. Hybrid method, the current most frequently used method, takes advantages of above two methods. It describes subordinate relations among different levels of parts and subassemblies in the multi-way tree and assembly relations in the assembly linkage graph. A certain type of temperature-humidity sensor for automatic weather station is displayed in Fig. 1, and its traditional relation model in Fig. 2. Intermediate nodes of multi-way tree represent subassemblies, and leaf nodes parts. The Edge between each two nodes describes their assembly relation. However, the traditional hybrid method does not take, complexity of the operations into account. It lacks reasonable constraints and simplification, which results in complicated relations at the same level and correrations across different levels. Its corresponding assembly sequences are also complex and lengthy. The situation is even worse when a reversible virtual process of assembly and disassembly occurs. Users have to make more control of camera view as to complete a large number of complicated and intricate virtual assembly operations. Thus, appropriate constraints have to be added into hybrid method to optimize its design according to practical needs.
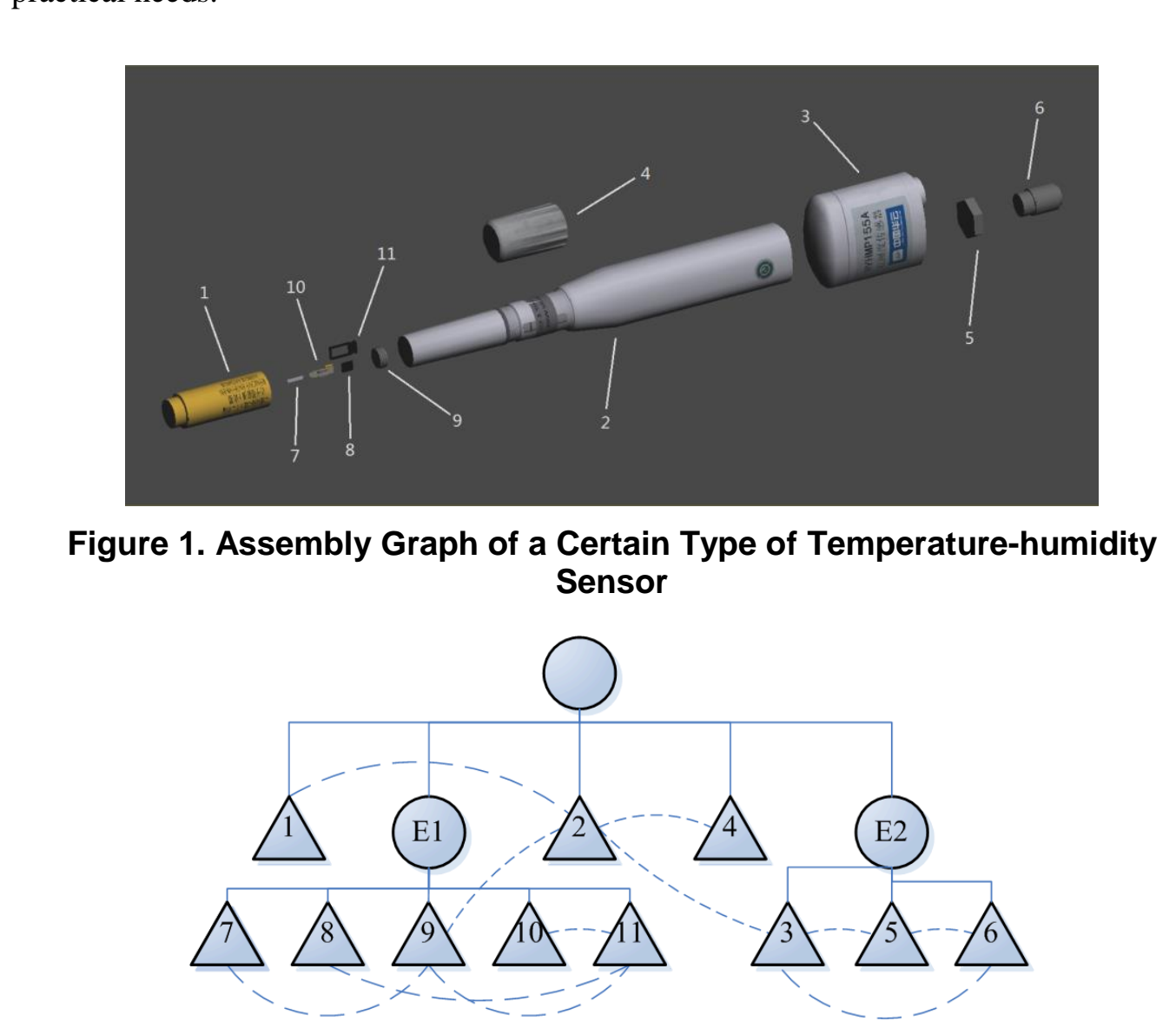

Figure 2. Traditional Assembly Relation Model of Temperature-humidity Sensor

The traditional virtual assembly relation models, based on hierarchical structure of parts and subassemblies, have complicated relations at the same level and correlations 
across different levels. These sophisticated assembly relations result in intricate and lengthy assembly sequences. Also, the traditional models do not take camera view control into account. When camera view follows the complex assembly sequences to adaptively adjust, missing camera view is prone to occur. The case is even worse especially when the traditional model applies in the description of reversible virtual assembly and disassembly. Thus, appropriate constraints have to be added into hybrid method to optimize its design according to practical needs.

\section{Optimized Virtual Assembly Relation Model}

\subsection{Establishment of Static Relation Tree}

The paper proposes an optimized virtual assembly relation model (optimized model) to meet needs of camera view control. The core concept of optimized model is base object which is set to simplify constraints applied in hierarchical structure of parts and subassemblies. A part, as an indivisible unit, is expressed as a leaf node in the relation tree. A subassembly is described as an intermediate node with multi-layer structure which can be divided into smaller parts at lower level. At every level of each subassembly, only one base object is defined as the foundation of any operation no matter whether assembly or disassembly. Parts and subassemblies, where direet connections among each other are not permitted, must be connected to corresponding base object of their own level. What is more, there are orderly sequence of constraints for parts and subassemblies at different levels during assembly and disassembly. Those at the upperleyel are prior to ones at the lower level in the sequence of disassembly and vice versa. Those at the same level can be operated in parallel. The optimized model is defined indetails as below.

Definition 1: leaf nodes of assembly relation tree represent parts, and intermediate nodes subassemblies. The edge between a partior subassembly and its base object reflects their connection status. Number 1 is labeled as connection, and number 0 as disconnection. Internal connections inside one subassembly are complete before it begins to be operated as a whole duing assembly and disassembly.

Definition 2: Suppose MT is one virtual operation of assembly or disassembly. Suppose $O$ is the sharing base object of part A and part B which compose the subassembly E. P is the base object of subassembly E. Virtual operations among parts, subassemblies and their hacing base object at the same level follow the rules as below.

1) Suppose there only exists A MT O and does not exist O MT A. The direction of assembly is assumed 0 be fixed. It is only permitted that a part or subassembly is installed to its base object.

2) Suppose there does not exist A MT B or B MT O. Unique base object is defined at each lerel. Assembly relations are not allowed other than the one between a part or subasembly and its base object. If there are more than one base objects at the same level, combrnation and redefinition may be in need, denoted by $\mathrm{A} \cup \mathrm{B}=\mathrm{E}$ and $\mathrm{P}=\mathrm{B}$.

3) Suppose there does not exist A MT O $\rightarrow$ B MT O. Parts and subassemblies sharing the same base object, which can be operated in parallel, do not have any operational order. If the order is necessary, relevant components are regarded as a new subassembly, denoted by $\mathrm{A} \cup \mathrm{B}=\mathrm{E}, \quad \mathrm{P}=\mathrm{A}, \quad \mathrm{B}$ MT $\mathrm{P}=\mathrm{E} \rightarrow \mathrm{E}$ MT O.

4) Subsequent operations MT of assembly and disassembly must comply with currently existent MT.

From the definitions already discussed, optimized model has evolved from traditional ones with better interpretation of assembly relation. There comes out a new idea that a 
new subassembly forms at upper level after parts or subassemblies are connected to their own base object at lower level. Static relation tree based on optimized model of temperature-humidity sensor is shown in Fig. 3. The square represents a part, and the cycle a subassembly. Triangle describes base object of each level inside a subassembly, and dotted line the assembly relations.

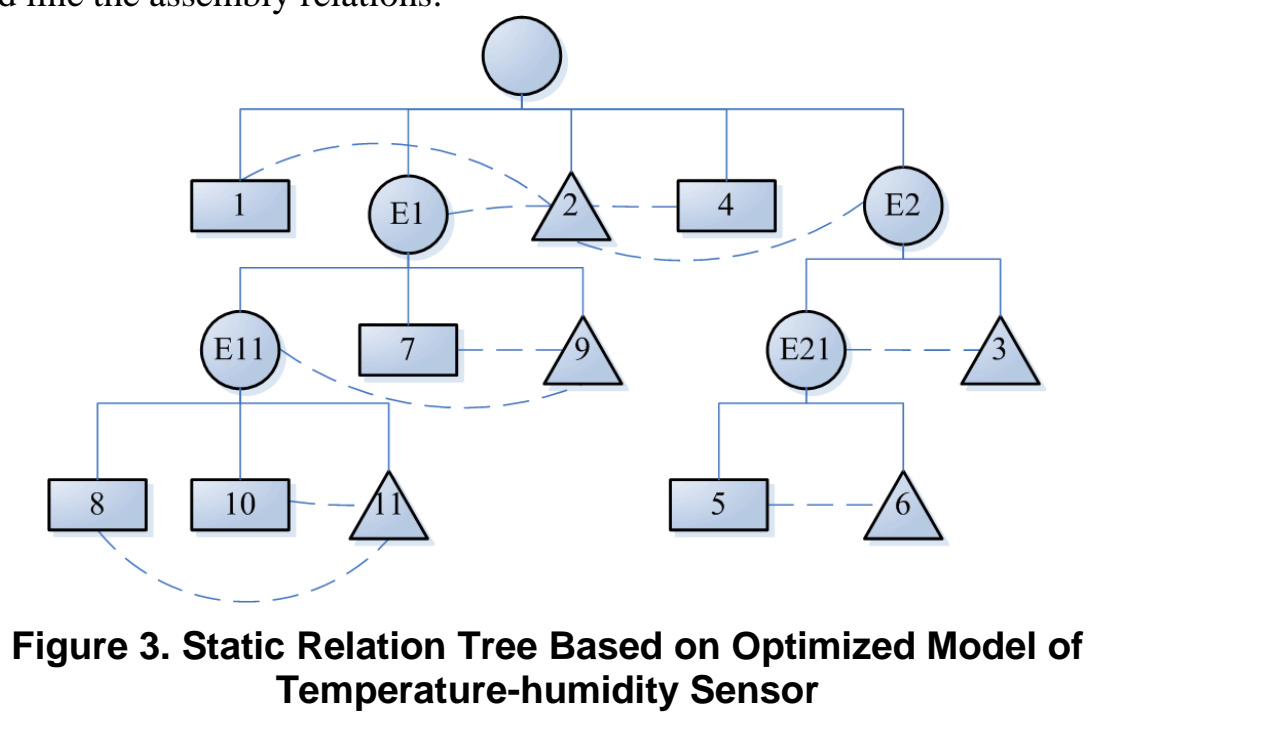

\subsection{Generation of Dynamic Relation Tree}

The static relation tree provides us with static structural relations among parts and subassemblies. It does not satisfy demands of assenbla and disassembly since the virtual operations continuously change. As users make vintual operations, the connection status among parts and subassemblies need to be updated in assembly linkage graph and the structure of relation tree has to be adjusted aue to real-time updated constraints. An example is given for dynamic relation tree. Subassembly E11, belongs to another subassembly E1, consist of part 8, part 10 and part 11 which is base object at the third level. When users choose installation, the subassembly E11 forms as a whole only after all relevant components are connected to their sharing base object (part 11). Then E11 is able to be link with yts base object 9 at upper level to form subassembly E1. When users choose uninstallation, the gase is similar and reserve operation is necessary. The subassembly E11 is not-complete if any related component cuts connection to its base object (part 11). This also leads to automatic disconnection between E11 and its base object 9 at upper level. Generation of dynamic relation tree for assembly and disassembly is shown respectively in Fig. 4 and 5. Some nodes in the solid line represent current existent components. Others in dotted line reflect incomplete subassemblies which are not displayed as a whole. 


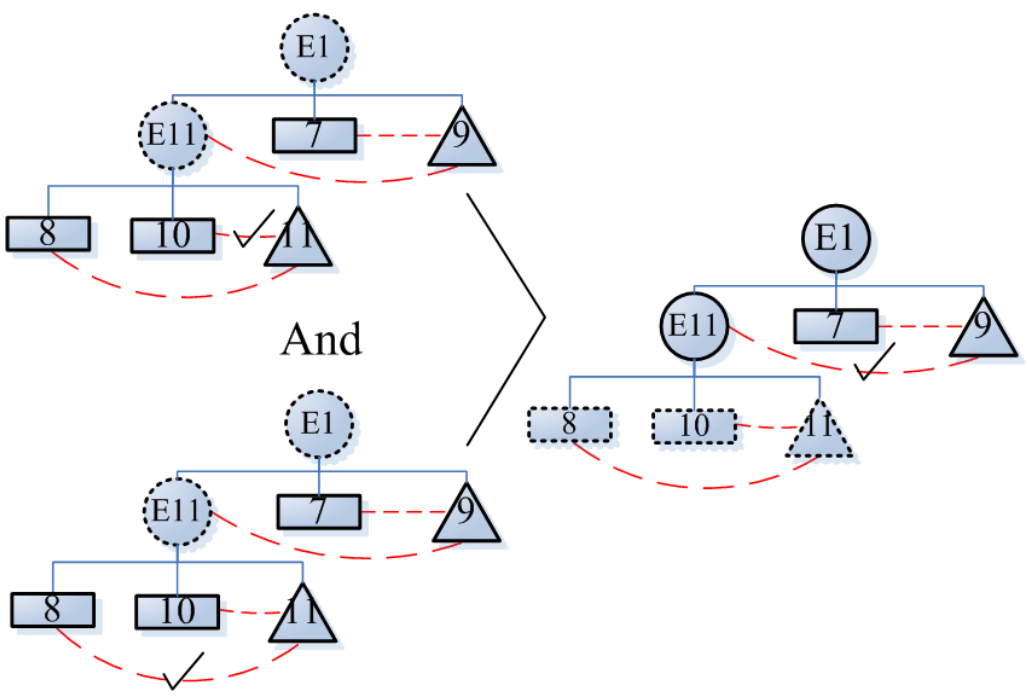

Figure 4. Dynamic Relation Tree of Assembly

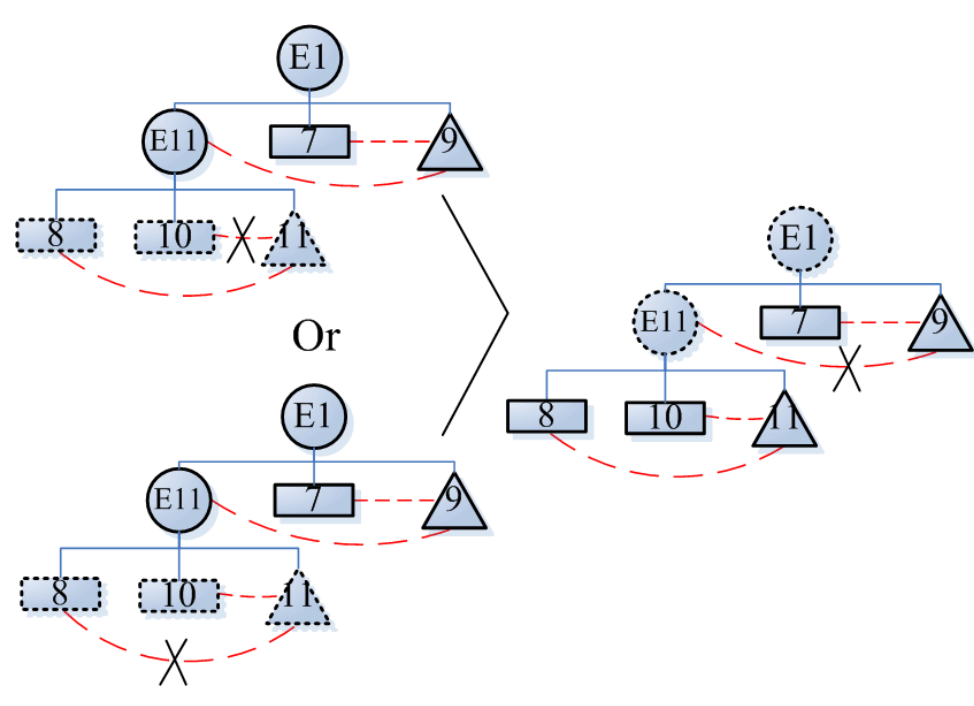

Figure 5. Dynamic Relation Tree of Disassembly

\section{Design of Adaptive Virtual Camera View Modes}

The optimized model discussed above, the basis of adaptive virtual camera view modes, sets up a hierarchical structure of parts and subassemblies in the reversible virtual process of assembly and disassembly. As the foundation of designed view modes, the optinized model gives an orderly and linear sequence for assembly and disassembly process. The virtual camera view modes are bound to focus on currently operated objects to avoid view distraction and wrong relocation of view backward because of translation and rotation operations. These modes provide users with practical guidance on correct operations of assembly and disassembly with precise control of its focus and range.

\subsection{Mathematical Model of Adaptive View Modes}

Suppose $\mathrm{E}$ is target object (TO), and $\mathrm{C}$ virtual camera. The $\mathrm{C}$ is designed to point to $\mathrm{E}$ and limit its movement on the dynamic virtual sphere of radius $\mathrm{R}$ which is the distance between $\mathrm{E}$ and its related operational object. The camera $\mathrm{C}$ is admitted to rotate only around latitudinal rotation axis $n 1$ and longitudinal rotation axis $n 2$ to ensure that $C$ is 
continuously focus on E. The principle schematic is shown in Fig. 6. The calculation process of adaptive view modes is demonstrated as follows.

(1)

$$
n \overrightarrow{1}=\mathrm{EC} \times n \overrightarrow{2}
$$

$$
n \overrightarrow{2}=(0,1,0)
$$

$$
\mathrm{R}=|\mathrm{EC}|
$$

(3)

$$
\overrightarrow{\mathrm{EC}}=\left(\mathrm{C} x-\mathrm{E} x, \mathrm{C} y-\mathrm{E} y, \mathrm{C}_{z}-\mathrm{E} z\right)
$$

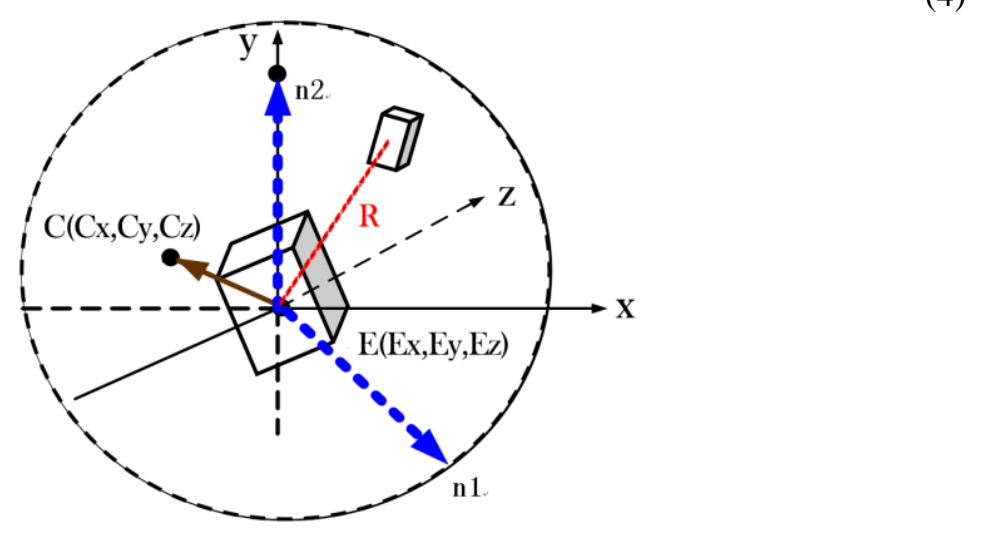

Figure 6. Mathematical Model of Adaptive View Modes

\subsection{Parameter Settings of Adaptive Mew Modes}

Suppose focus $\mathrm{O}$ reflects the center of virtual view sphere, and radius $\mathrm{R}$ field of view. The triangle describes base object $\mathrm{B}$, the oycle target object $\mathrm{E}$, and the square other objects. The adaptive view modes have two typical cases respectively for assembly and disassembly.

4.2.1. Assembly view mode: Assiembly view mode continuously sets base object B as focus $\mathrm{O}$ and adjust the field of wiew along with the distance between target object $\mathrm{E}$ and its base object B. When E and B get closer, the mode zooms in and vice versa. The starting point of this mode is to offer users convenient and brief operational experience in assembly process. The constraints of optimized model assuming that a part or subassembly can only beinstalled into its base object B have simplified the design. When the assembly of a paft or subassembly is done, the focus concentrates on base object $\mathrm{B}$ and the field of view covers all parts and subassemblies with sharing base object B and incomplete assembly status. Users follow the assembly mode to easily install other components What's more, one special case needs to be considered when all parts and subassemblies whose base object is B are entirely completed after installation, a new base obje t, searched along former base object B's parent node direction in dynamic relation tree and found incompletely installed, is set as a new view focus. The assembly mode leads to two cases of view focus $\mathrm{O}$ and field of view $\mathrm{R}$ as shown in Fig. 7. The red solid line represents the current field of view, and the red dashed line the former one. 


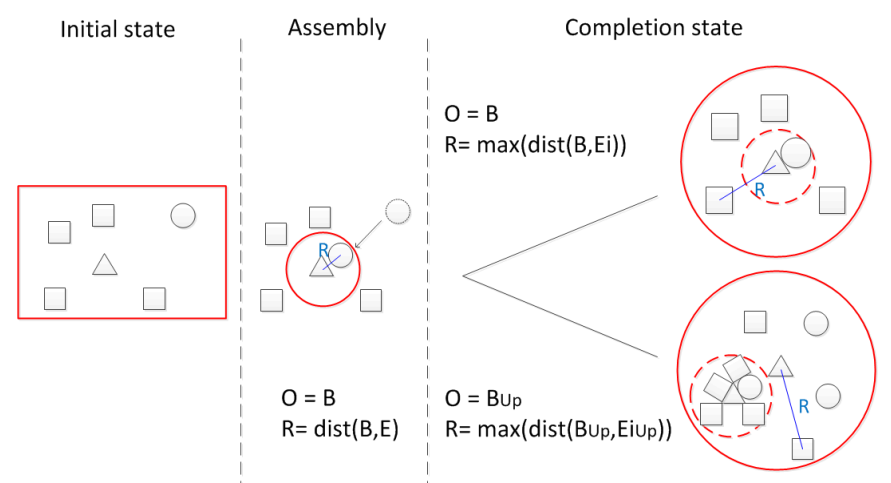

Figure 7. Assembly View Mode

4.2.2. Disassembly view mode: The disassembly view mode takes the middle point between target object $\mathrm{E}$ and its base object $\mathrm{B}$ as the view focus, different from assembly view mode. The field of view zooms out to observe the variance of distance and location of $\mathrm{B}$ and $\mathrm{E}$. If $\mathrm{E}$ is a subassembly and its installation is done, the camera focuses on $\mathrm{E}$ and the radius $\mathrm{R}$ equals to the distance between $\mathrm{B}$ and $\mathrm{E}$. This allows users not only to take closer look at the subassembly $\mathrm{E}$ which can be uninstalled as many parts at lower level, but also to uninstall other components whose base object is also B. if E. is a part and its disassembly is over, the camera focuses on curren base object $B$ and its range covers all components whose disassembly is not done and base object is also B. Users may follow the disassembly mode to uninstall other parts and subassemblies more easily because all components closely related to part $\mathrm{E}$ arencontained inside the field of view. If all components sharing the same base object B are entirely, taken apart, a new view focus is searched along B's parent node directionin dynamic relation tree until a new base object is found incomplete disassembly In a word, disassembly view mode results in three parameter groups of $\mathrm{O}$ and $\mathrm{R}$ as shown in Fig. 8. The red solid line represents current field of view, and the dotted line the former one.

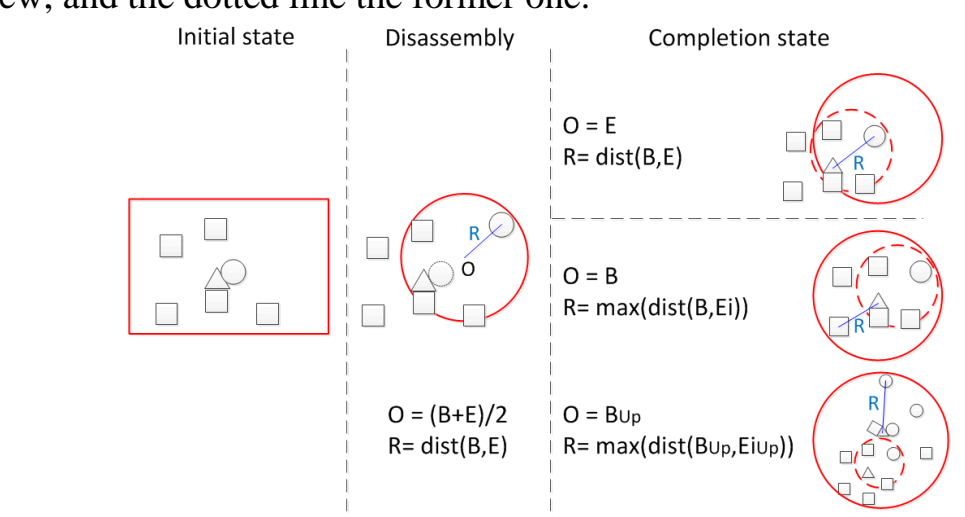

Figure 8. Disassembly View Mode

\section{Implementation of Adaptive Virtual Camera View Modes}

The main advantage of adaptive virtual camera view modes is that the view continues to follow and display the user's intention by dynamically setting parameters of camera such as focus and radius. The optimized model discussed above provides these view modes with brief design and effective implementation which brings lower difficulty and higher efficiency of operations. The workflow shown in Fig. 9 is divided into several main steps as follows.

Step 1: Start adaptive virtual camera view modes and wait for mouse events. 
Step 2: Determine whether the object in the current mouse event is a base object. If so, the view mode will change into special settings. Otherwise it goes to judgment on type of operations including assembly and disassembly.

Step 3: Decide whether the user plans to install or uninstall the part or subassembly picked up by mouse according to the value of edge between the component and its base object in the optimized model. If the value is equal to zero, it means separation of two objects and assembly view mode will be adopted. If the value equals one, the two objects connect each other and disassembly view mode will be automatically switch to.

Step 4: Update constraint conditions and parameter settings of the optimized model in real time as user operations go on.

Step 5: Reset parameter values of camera view including focus $\mathrm{O}$ and radius $\mathrm{R}$ dynamically according to completion of operations when user releases the mouse. When assembly of the object picked up by mouse is done, new parameter values will be generated. What's more, we need to decide whether the subassembly which the object belongs to has been installed as a whole according to the values of all edges connecting to the object's base object. If these value all equal one, adjustment of camera view will be made. When disassembly is done, we reset different parameter values based on completion of operations with the judgment that the picked-up object is a part or a subassembly.

Step 6: Enter the loop and wait for the next mouse event.

Step 7: Stop adaptive virtual camera view modes when userchoose to exit.

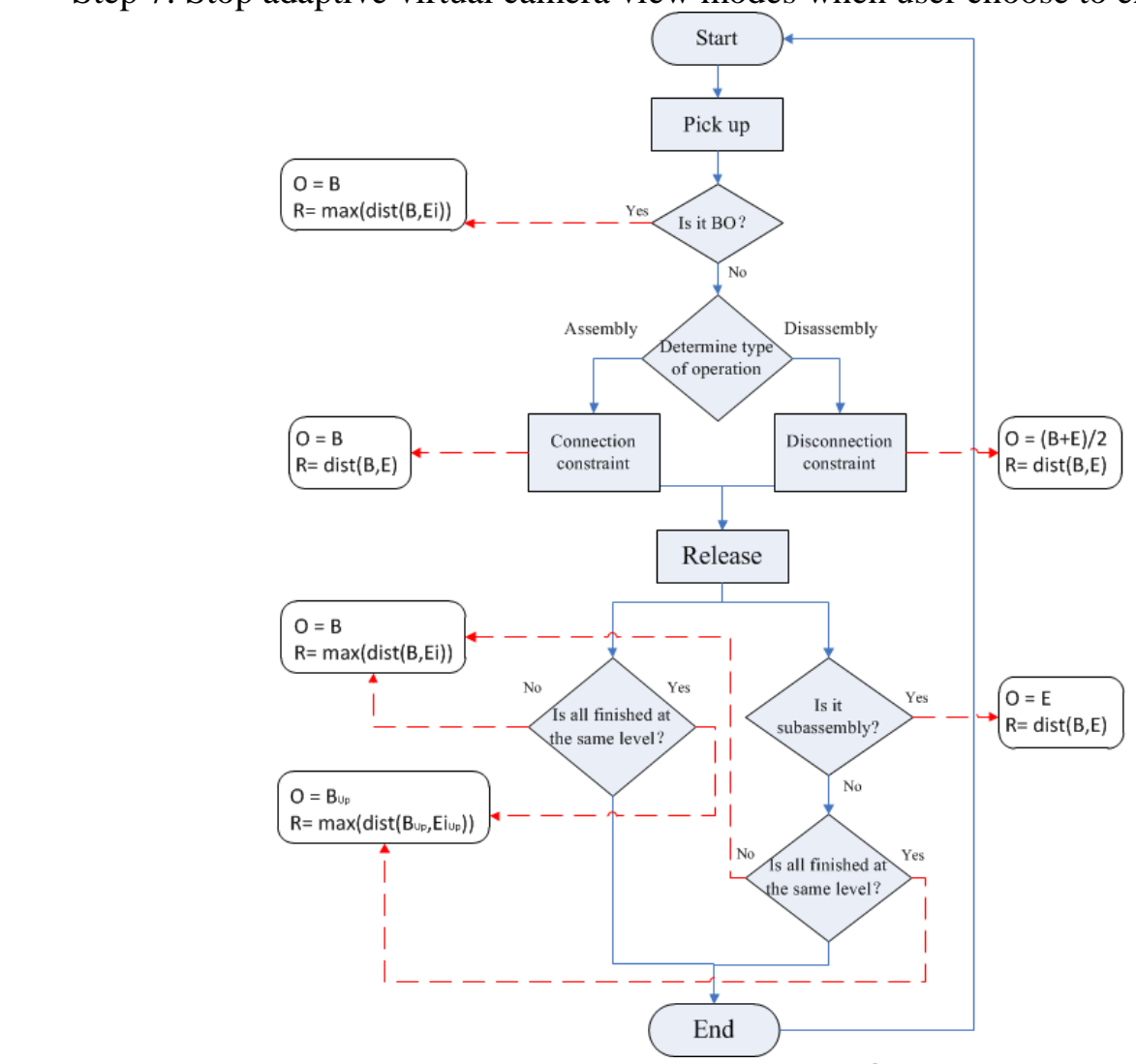

Figure 9. Workflow of Adaptive Virtual Camera View Modes 


\section{Experimental Evaluation}

To evaluate the efficiency of the adaptive virtual camera view modes, we conducted an evaluation including twenty four users whose age range is between 20 and 40 (mean = 27.04 , standard deviation $=4.63$ ). These users were divided into two groups each of which consists of 12 persons. One adopting the adaptive virtual camera view modes worked as experimental group, and the other without adaptive modes acted as control group. The objective of this evaluation was to verify whether users were able to reduce their operations by using adaptive camera view modes. According to features of virtual camera control, four key indexes were chosen to describe users' performance during their virtual assembly operations. The first one is completion time which represents users' operational maturity. The following three features are cumulative total of camera view operations, namely translation, rotation and scale, which reflect operational efficiency of camera view.

As shown in Table 1, the columns of "with" and "without" compared mean values of four features with or without adaptive modes. The last column reflected $\mathrm{p}$ value of a paired two-sample T-test between two groups. According to the statistical wesults, the completion time and cumulative total of translation with a p-value lower than $0.05 \mathrm{had}$ significant correlation. In other words, these two features could be used to describe the experimental results. After careful comparison of two groups, one conclusion was reached. Adaptive camera view modes enabled users to make fewer operations with the same effect. It showed that the adaptive virtual camera view allowed users to experience better control of camera view and more efficient operations.

Table 1. Test Results for Exxoenimentaland Control Groups

\begin{tabular}{|l|c|c|c|}
\hline \multicolumn{1}{|c|}{ Feature } & With & pithout & \\
\hline Completion time (sec) & 176.54 & 213.81 & 0.05 \\
\hline Translation (times) & 11 & 28 & 0.01 \\
\hline Rotation (times) & 14 & 17 & 0.25 \\
\hline Scale (times) & 7 & 6 & 0.61 \\
\hline
\end{tabular}

\section{Conclusion}

The adaptive virtual camera yiew modes discussed in this paper can be also applied in many other fields, such as product design, product exhibition and staff training. It helps to shorten product design cycere, reduce operational risks and cut costs. Especially in the training, it solves the traditional problem of hardly interpreted internal structure and working principles of complicated instruments. With the adaptive modes, users can make deep and careful observation of internal or shielded parts, and follow its guidance on correct operational flow, which significantly improve training efficiency. As future work, it would be interesting to investigate how to enhance users' experience. For example, the cooperation between lights and camera view is worthy of further study to obtain better visual effects.

O erall, we have focused on the control of virtual camera view which is an always gnored but unavoidable problem in virtual assembly system. The optimized model in this paper supposes appropriate constraints which simplify complicated assembly and disassembly of sophisticated equipment. The newly designed view modes based on optimized model have set up hierarchical virtual assembly relations. On account of appropriate constraints and designs, the view modes have broken through the technical barrier of hard control of 3D virtual camera view, which have improved user experience and operation efficiency. 


\section{References}

[1] M. Schenk, S. Straßburger, H. Kissner, "Combining Virtual Reality and Assembly Simulation for Production Planning and Worker Qualification," Proceedings of the International Conference on Changeable, Agile, Reconfigurable and Virtual Production (CARV 2005), eds. M. Zaeh and G. Reinhart, pp. 411-414, September 2005.

[2] John E. Brough, Maxim Schwartz, Satyandra K. Gupta, Davinder K. Anand, Robert Kavetsky, Ralph Pettersen, "Towards the development of a virtual environment-based training system for mechanical assembly operations," Virtual Reality, vol. 11, no. 4, pp. 189-206, 2007.

[3] Wesley M A et al, "A geometric modeling system for automated mechanical assembly," IBM Journal of Research and Development, vol. 24, no. 1, pp. 64-74, 1980.

[4] Mike Oren, Patrick Carlson, Stephen Gilbert, Judy M. Vance, "Puzzle assembly training: Real world vs. virtual environment," IEEE Annual International Symposium - VR, pp. 27-30, 2012.

[5] LI Chang- guo, ZHU Fu- quan, TAN Liang, YANG Chun, "Research on Development Approaches of Virtual Experiment Based on 3D and Virtools Technologies," Computer Engineering and Applications, vol. 42, no. 31, pp. 84-87, 2006.

[6] ZHAO Hai-hui, MENG Chui-cheng, SHU Qi, "The Application of VR Design and Simulation in Oil Machinery Design,” Journal of Engineering Graphics, vol. 28, no. 4, pp. 1-5, 2007.

[7] WANG Wei-da TURNER J U,SUBRAMANIAM S,GUPTA S, "Constraint representation and reduction in assembly modeling and analysis," IEEE Transactions on Robotics and Automation, vol. 8, pp. 741-750, December 1992.

[8] Lee K, Gossard D C, "A hierarchical data structure for cepresenting assernblies: Part 1," Computer-Aided Design, vol. 17, no. 1, pp. 15-19, 1985.

[9] Thomas J P, Nissanke N, Baker K D, “Boundery models for assembly knowledge representation,” IEEE Transactions on Robotics and Automation, vol. 12, no. 2, pp.302-312, 1996.

[10] Wilson R H, Latombe J C, "Geometric reasoning about mechanical assembly," Artificial Intelligence, vol. 71, no. 2, pp. 374-396, 1994.

[11] WU Dian-liang, Yang Run-dang, MA Deng-zhe. FAN Xiu-min, Virtual Assembly System and the Enabling Technologies," Journal of Shanghar Jiaotong University, vol. 38, no. 9, pp. 1539-1543, September 2004.

[12] Homen de Mello, Sanderson A C “Computer-Aided mechanical assembly planning," IEEE Transactions on Robotics and Automation, vol. 7, no 2, pp. 228 240, 1991.

[13] SHI Miao, TANG Shuo-fei, LN Ming-shu, “A review of assembly sequences planning," Computer Research and Development, vol. 31, no. 6, pp. 30-31, 1994.

[14] YAO Jun, NING Ru-xin, WANG Xin-ýong, "Research of virtual reality based assembly planning," Chinese Journal of Mechanical Engineeting, vol. 38, no. 8, pp. 130-134, 2002.

[15] WANG Jun-feng, MU Ji-hong, ZHONG Yi-fang, "Collaborative Assembly Planning System under Web Environment," Computer Integrated Manufacturing Systems, vol. 10, no. 1, pp. 83-88, January 2004.

[16] Zhong Y M, Wolfgang M W, Ma W Y, "Incorporating constraints into a virtual reality environment for intuitive and precise solid modelling," Sixth International Conference on Information Visualisation(1v'02), pp 38?-398, 2002.
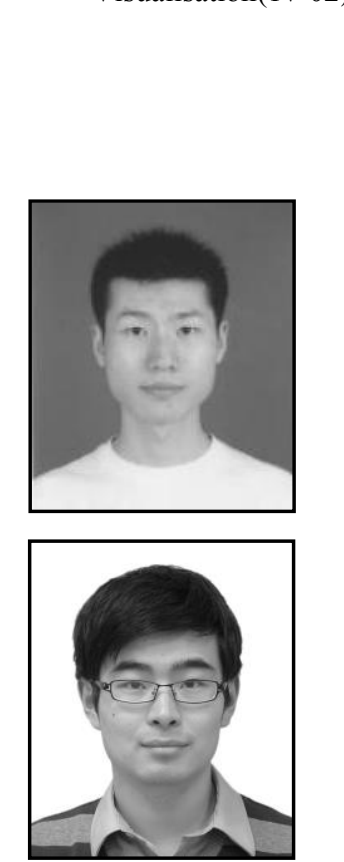

\section{Authors}

Rui Yang, is with the China Meteorological Administration Training Centre, China Meteorological Administration, Beijing, P.R. China (e-mail: yangr@cma.gov.cn).

WenJie Fan, is with the China Meteorological Administration Training Centre, China Meteorological Administration, Beijing, P.R. China (e-mail: fanwj@cma.gov.cn). 